\title{
A New Fourier-Based Approach to Measure Irregularity of Breast Masses in Mammograms
}

\author{
Gensheng Zhang, Sung Shin, Wei Wang \\ Department of Electrical Engineering and Computer Science \\ South Dakota State University \\ Brookings, SD, 57006, USA \\ gensheng.zhang@jacks.sdstate.edu \\ \{sung.shin,wei.wang\}@sdstate.edu
}

\author{
Carrie Hruska ${ }^{1}$, Hyung D. Choi ${ }^{2}$ \\ ${ }^{1}$ Department of Radiology, Division of Medical \\ Physics Mayo Clinic \\ Rochester, MN, 55905, USA \\ carrie.hruska@mayo.edu \\ ${ }^{2}$ Radio Technology Research Department \\ Electronics and Telecommunications Research \\ Institute (ETRI) Daejeon 305-700, South Korea \\ choihd@etri.re.kr
}

\begin{abstract}
Morphologic appearance is one of intuitive diagnosis factors of mass lesions in breast imaging, and irregular shape is one of the most frequent appearances for malignant masses. Thus, an effective measure of morphological irregularity will provide a helpful reference to determine malignancy of breast masses. In this paper, a new measure based on Fourier Transform, named Fourier Irregularity Index $\left(\mathrm{F}_{\mathrm{II}}\right)$, was developed to provide a reliable malignant/benign classification factor. The experiment was conducted with 418 breast masses, including 190 malignant cases and 218 benign cases. Performance was assessed and compared among various methods using Receiver Operating Characteristics (ROC) analysis. The proposed measure in this study achieved malignant/benign classification accuracy of $96 \%$ with an area $\left(\mathrm{A}_{\mathrm{z}}\right)$ of 0.99 under the receiver operating characteristics (ROC) curve, which outperformed typical traditional approaches, such as Compactness (accuracy of $90 \%, \mathrm{~A}_{z}$ $=0.96$ ), Fractal Dimension (accuracy of $90 \%, A_{z}=0.95$ ), Fourier Factor (accuracy of $90 \%, \mathrm{~A}_{\mathrm{z}}=0.97$ ), and Fractional Concavity (accuracy of $75 \%, \mathrm{~A}_{\mathrm{z}}=0.65$ ).
\end{abstract}

\section{Categories and Subject Descriptors}

I.4.7 [IMAGE PROCESSING AND COMPUTER VISION]: Feature Measurement-Feature representation, Size and shape, Texture.

\section{General Terms}

Measurement; Experimentation.

\section{Keywords}

Irregularity Measure, Fourier Irregularity Index, Shape Factor.

\section{INTRODUCTION}

Breast cancer is one of most commonly diagnosed cancer among American women. About 1 in 8 U.S. women (about 12\%) will develop invasive breast cancer over the course of her lifetime[1]. It is believed that mammography is one of the most effective breast screening techniques that achieves the mortality reduction

Permission to make digital or hard copies of all or part of this work for personal or classroom use is granted without fee provided that copies are not made or distributed for profit or commercial advantage and that copies bear this notice and the full citation on the first page. To copy otherwise, or republish, to post on servers or to redistribute to lists, requires prior specific permission and/or a fee.

RACS'12, October 23-26, 2012, San Antonio, TX, USA.

Copyright 2012 ACM 978-1-4503-1492-3/12/10 ..\$15.00. by early detection of breast cancer [2]. However, mammographic interpretation is a complicated task requiring considerable domain expertise. Earlier studies show that, $10 \%-25 \%$ of tumors are missed by the radiologists[3].

It is usually interpreted as the evidence of breast tumor that a dense mass presents in the mammogram. Morphologic appearance is one of criteria to classify the type of masses or tumors. In describing mass shapes, substantial agreement between breast radiologists was seen when the mass shape was characterized as irregular (the Cohen's kappa coefficient value is 0.68) [4]. A nonuniformity of growth often leads to an irregular shape, often seen in carcinomas[5]. Thin, irregular calcifications with linear or branching morphology suggest filling of the lumen of a duct involved irregularly by breast cancer. A typical benign mass is round and smooth with a well-defined boundary. On the contrary, a typical malignant tumor is in irregular shape and rough with a blurry boundary. In medical field, most of time, it is important to derive medical properties from those shape characteristics instead of absolute shape. In other words, it is possible to distinguish benign masses from malignant tumors by examining their shape characteristics, or morphological irregularity.

A few studies have examined the possibility of classifying breast masses based on the irregularity exhibited in their contour. As early as in [6-8], compactness measure is used to characterize the diffused or compact nature of calcifications, rather than roughness. A circumscribed/speculated classification rate of $92.3 \%$ can be achieved by measuring the compactness of shapes [9]. However, compactness is limited to certain circumstance due to its sensitivity to noise along the border. For malignant tumors typically possess both concave and convex segments as well as microlobulations and prominent spicules, the fractional concavity $\left(f_{c c}\right)$ is used as a feature in pattern classification experiments [10]. Besides its high sensitivity $(88.5 \%), f_{c c}$ resulted in a poor specificity of $60.7 \%$ because it failed to look into the characteristics of the spicules in terms of their depth and narrowness. As an important tool of characterizing complex geometric form, fractal dimension (FD) is widely used as a shape feature. For example, FD is calculated to characterize the complexity of regions of interest (ROIs) in mammograms by Guo et al. [11]. Rangayyan et al. investigated four methods to compute the FD of the contours of breast masses, including the ruler method and the box counting method applied to $1 \mathrm{D}$ and 2D representation of the contour [12]. While FD methods themselves showed relatively good performance, the result also showed FD and fractional concavity $\left(f_{c c}\right)$ can compensate for each other's weakness. However, the normalization procedure proposed in [12] 
may cause loss of details along the border. The other drawback of FD is that it is insensitive to large structure indentations and protrusions [13]. Because of its popularity, Fourier Transform (FT) was introduced in image processing many years ago. One of interesting irregular measure based on FT, named Fourier Factor (FF)[14], believed that FF would give a small value if more energy is distributed in the high-frequency part (which means the shape has a rough boundary). The reported result show FF correctly classified 54 out of 64 benign calcifications $(84.38 \%)$, and correctly classified 67 out of 79 malignant calcifications $(84.81 \%)$. In other biomedical fields, contour irregularity is also considered as a diagnosis reference. Lee et al. $[13,15]$ investigated several shape descriptors, such as compactness, FD and irregularity index, to study the irregularity of melanocytic lesion contour. Kikuchi et al. believes fractal geometry can be used to describe the pathological architecture of ovarian tumors[16].

Unfortunately, these approaches are either insensitive to structural features which leads to a poor sensitivity, or too sensitive to noises which results in a high false positive rate. The aim of our study is to present a novel effective method, named Fourier Irregularity Index $\left(\mathrm{F}_{\mathrm{II}}\right)$, to analyze the irregularity of breast mass contours. The experiment results show that it is possible to integrate our method into a computer-aided diagnosis (CAD) system as a reference to automatic diagnosis.

The remainder of this paper is structured as follows. The following section details the algorithm for our new measures, named Fourier Irregularity Index $\left(\mathrm{F}_{\mathrm{II}}\right)$. The constituent of testing data set, the evaluation protocol, as well as the methods used to compare with our approach are briefly described in Section 3. Section 4 discusses our experiment results and Section 5 concludes our study with a summary.

\section{FOURIER IRREGULARITY INDEX}

In order to measure irregularities of shapes, finding an appropriate shape descriptor is the first step. The shape descriptor we used in our study is called origin distance descriptor, which is defined as the radial distance from each contour point to the origin as a function of the index of the contour point. By examining the descriptor carefully, an interesting phenomenon can be observed that the origin distance changes more and faster if the shape has more roughness along the contour. In other words, the series has more high frequency parts in its frequency domain representation than those derived from smooth, round shapes.

As one of the most popular and important time-frequency analytical tools, Fourier Transform is employed to investigate such phenomena in details. Before transforming origin distance descriptor into Fourier series, a simple normalization procedure is performed to the original coordinates: move the origin to left-top corner of the contour, as given in following equation:

$$
O D S^{\prime}=\left[p_{0}, p_{1}, \ldots, p_{k}, \ldots, p_{N-1}\right]
$$

where $p_{k}$ is the kth contour point, and

$$
\begin{aligned}
& p_{k}=\left(x^{\prime}{ }_{k}, y^{\prime}{ }_{k}\right)=\left(x_{k}-\min _{i} x_{i}, y_{k}-\min _{i} y_{i}\right) \\
& \left(x_{k}, y_{k}\right) \text { is the original kth contour point }
\end{aligned}
$$

Suppose that $\mathrm{N}$ radial distances are derived from the normalized origin distance descriptor. The shape then may approximately be written as a time series $r$ :

$$
r=\left[r_{0}, r_{1}, \ldots, r_{k}, \ldots, r_{N-1}\right]
$$

where

$$
r_{k}=\sqrt{x_{k}^{\prime 2}+y_{k}^{\prime 2}}
$$

Obviously, the sequence is periodic with each traversal (clockwise or counter-clockwise) of the complete boundary. According to the discrete-time Fourier Transform (DTFT) formula [17], series $r$ can be transformed into its frequency domain representation:

$$
\begin{gathered}
R=\left[R_{0}, R_{1}, \ldots, R_{k}, \ldots, R_{N-1}\right] \\
\text { where } \quad R_{k}=\sum_{n=0}^{N-1} r_{n} \cdot e^{-i 2 \pi \frac{k}{N} n} k=0,1, \ldots N-1
\end{gathered}
$$

The inverse discrete Fourier Transform (IDFT) is given by:

$$
r_{k}=\frac{1}{N} \sum_{n=0}^{N-1} R_{k} \cdot e^{+i 2 \pi \frac{k}{N} n}
$$

As a round mass has high possibility to be benign, these shapes are defined as regular shapes, or the least irregular shapes, which should have a small irregularity. On the contrary, shapes are defined as irregular shapes if they change its origin radial distance rapidly. In order to meet these basic conceptions, the Fourier Irregularity Index $\left(\mathrm{F}_{\mathrm{II}}\right)$ is defined as:

$$
F_{I I}=1-\frac{\sum_{k=1}^{N / 2} \Re_{k} / k}{\sum_{k=1}^{N / 2} \Re_{k}}
$$

where

$$
\mathcal{R}_{k}=\left|\frac{R_{k}}{R_{1}}\right| n=1,2 \ldots N / 2
$$

According to the definition, energy $\boldsymbol{\mathcal { R }}_{k}$ will be distributed in different frequencies with respect to the radial distance variations. As we can see from the definition, the energy $\boldsymbol{\mathcal { R }}_{k}$ of highfrequency parts will be diluted due to the division by $k$. In other words, $\mathrm{F}_{\mathrm{II}}$ would have a big value if more energy distributed in high-frequency parts, which is true for rough shapes. Take case A (A_1284_1.LEFT_MLO in DDSM) and case B (B_3022_1.RIGHT_MLO in DDSM) as examples, case A is diagnosed as a benign breast mass with an oval shape, and case $\mathrm{B}$ as malignant breast mass with an irregular shape (architectural distortion). From Figure 1, we can see case B (dashed line) has more energy distributed in high frequency parts than case A (solid line) does. It matches the fact that contour $\mathrm{B}$ is rougher than contour A.

We can summarize some characteristics of $F_{\text {II }}$ from above analysis:

1) The range of $F_{I I}$ lies in $[0,1]$;

2) $F_{\text {II }}$ yields small values for regular shapes like round or oval shapes, while big values for irregular shapes;

3) The more energy distributed in high frequency components, the more irregular the shape would be, the larger the $\mathrm{F}_{\mathrm{II}}$ value would be.

\section{EVALUATION METHODOLOGY}

\subsection{Testing data inputs}

In this section, the concept developed in this study is applied to a set of mammograms provided by the Digital Database for Screening Mammography (DDSM) [18]. There are about 2,500 


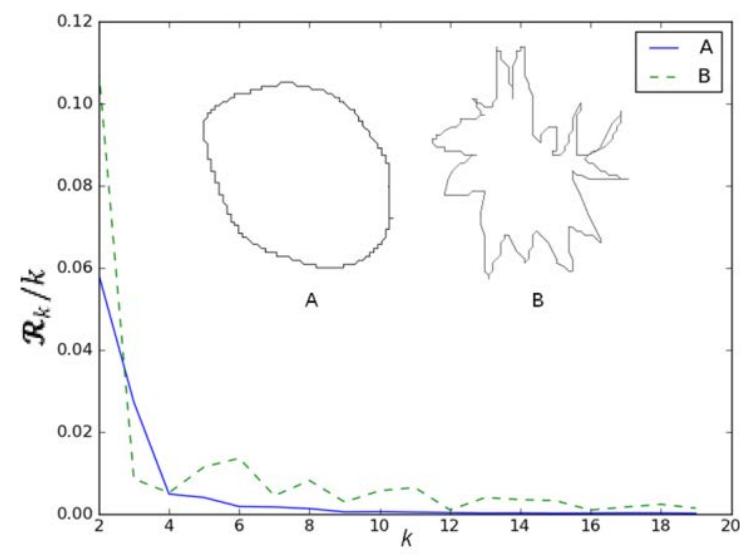

Figure 1 An example of energy distribution. More energy distributed in high frequency parts in case $B$ (dashed line) than case A (solid line), matches the fact that B has a rougher boundary than A does.

studies in the database. Each study includes two images of each breast. Associated pixel-level "ground truth" information are available including the locations and types of suspicious regions, as well as patient information and image information. Abnormalities of abnormal cases are described in "OVERALY" files. Each abnormality has information of the lesion type, the assessment, the subtlety, the pathology and at least one outline. Besides mentioned information, the outlines for the suspicious regions are also derived from markings made on the film by an experienced radiologist. Each boundary is encoded in Freeman Chain Code of Eight Directions[19]. To be simple, only three types of shapes are included in the testing data set: round, oval and irregular. Totally 418 mass cases are investigated in our study, including 190 malignant cases and 228 benign cases.

\subsection{Evaluation protocol}

As a fundamental tool for diagnostic test evaluation, the Receiver Operating Characteristic (ROC) curve analysis is widely used in medical field. An ROC curve is a plot of a test's sensitivity (plotted on the vertical axis), versus its False-Positive Rate (FPR), or 1-specificity (plotted on the horizontal axis)[20], where sensitivity and specificity are defined in Equation 6 and 7, respectively. Each point on the curve represents the sensitivity and FPR at a different decision threshold. The area under the curve, usually referred as the $A_{z}$ index, is an accepted way of evaluating diagnostic performance[21], where larger $A_{z}$ denotes higher performance. Perfect diagnostic accuracy is achieved if the ROC curve has an $A_{z}$ index of 1.0 .

$$
\begin{aligned}
& \text { sensitivity }=\frac{T P S}{T P S+F N S} \\
& \text { specificity }=\frac{T N S}{T N S+F P S}
\end{aligned}
$$

where TP stands for true positive, FN for false negative, TN for true negative and FP for false positive. The sensitivity specifies the probability that the diagnostic test is negative for disease for a patient who truly does not have the disease, while the specificity specifies the probability that the diagnostic test is negative for disease for a patient who truly does not have the disease.

\subsection{Methods}

Four previously reported methods for analysis of breast messes in mammograms were implemented to perform a comparative analysis of $F_{I I}$ as a factor for classification purpose. These four approaches are briefly described in the following subsections.

\subsubsection{Compactness}

Compactness is developed to measure how efficiently a contour encloses a given area. The following equation gives the definition of the normalized measure of Compactness (C)[22]. Since circles are the most compact 2D shapes, they have the smallest compactness of zero. A high compactness usually indicates the contour contains much roughness along the boundary, which may imply the malignance of a suspicious area[23].

$$
C=1-\frac{4 \pi A}{P^{2}}
$$

where $\mathrm{A}$ is the enclosed area and $\mathrm{P}$ is the perimeter of the contour.

\subsubsection{Fractal dimension}

Fractal Dimension (FD) is a measure of the roughness or jaggedness of a border or a surface. The FD of a contour L can be estimated by using either box-counting method or ruler method. Box-counting method counts the number of $r \times r$ grids containing the contour after placing the grids over the contour. The relationship between FD, denoted by $\mathrm{D}$, and the number of grids $\mathrm{N}$ is expressed as:

$$
N=\epsilon r^{-D}
$$

where $\varepsilon$ is a constant.

Equation 9 can be expanded to:

$$
\log \frac{1}{N}=D \times \log r-\log \epsilon
$$

Thus, $\mathrm{D}$ is the slope of above equation, which can be computed by curve fitting techniques, such as the least square. Ruler method is similar to box-counting method. Instead of placing difference sizes of grids over the contour, ruler method measure the contour by different lengths of rulers. The FD is obtained from the linear slope of the plot of the log of measured length vs. the log of the ruler size (measuring unit). Only ruler method is investigated in this paper for its simplicity and efficiency. Our implementation has a close value to theoretical one when we apply it to Koch's snowflake (1.2593 vs. 1.26).

\subsubsection{Fourier factor}

Fourier Factor (FF) is a measure of how the energy is distributed in frequencies[14]. Basically a small value of FF indicates more energy is distributed in the high-frequency parts, which implies the truth for shapes with rough boundaries. The FF is derived from Fourier Transform of the coordinates of the contour pixels, as follows:

$$
F F=\frac{\sum_{k=-\frac{N}{2}+1}^{N / 2} \frac{\|N F D(k)\|}{|k|}}{\sum_{k=-\frac{N}{2}+1}^{N / 2}\|N F D(k)\|}
$$

where

$N F D(k)=\left\{\begin{array}{c}0 ; \quad k=0 \\ (A(k)) /(A(1)) ; k=1,2, \ldots, N / 2+1 \\ A(k+N) / A(1) ; k=-1,-2, \ldots,-N / 2+1\end{array}\right.$ 


$$
\begin{gathered}
A(k)=\frac{1}{N} \sum_{j=0}^{N-1} Z_{j} e^{-i \frac{2 \pi k}{N} j} \\
Z_{j}=x(j)+i \cdot y(j) \\
(x(j), y(j)) \text { is the } j^{\text {th }} \text { point in the contour }
\end{gathered}
$$

\subsubsection{Fractional concavity}

Fractional concavity $\left(f_{c c}\right)$ is the measure of the portion of the cumulative concave length $\left(C C_{l}\right)$ to the total boundary length [10], which is based on polygon approximation method. Intuitively, regular shapes, which usually are a factor of being a benign mass, have fewer portions of concave segments than irregular shapes (usually malignant).

\section{RESULTS AND DISCUSSION}

For each breast mass in the testing data set, we computed the Compactness (C), Fractal Dimension (FD), Fourier Factor (FF), Fractional Concavity $\left(f_{c c}\right)$ and Fourier Irregularity Index $\left(\mathrm{F}_{\mathrm{II}}\right)$. The performance of these methods is validated use ROC methodology. As one of indicator of performance in ROC analysis, the area $\left(A_{z}\right)$ under the ROC curve is computed using trapezoidal rule. The highest accuracy (the ratio of the number of correctly classified cases to the total number of cases) was also recorded during the analysis.

Table 1 Evaluation of classification results with 418 mammograms (190 malignant and 228 benign) by various feature studied: Compactness (C), Fractal Demension (FD), Fourier Factor (FF), Fractional Concavity $\left(f_{c c}\right)$, Fourier Irregularity Index $\left(\mathrm{F}_{\mathrm{II}}\right)$

\begin{tabular}{l|lllll}
\hline & TP/FN/FP/TN & Sensitivity & Specificity & Total & $\boldsymbol{A}_{z}$ \\
\hline C & $171 / 23 / 19 / 205$ & 0.881 & 0.915 & 0.90 & 0.96 \\
FD & $157 / 11 / 33 / 217$ & 0.935 & 0.868 & 0.90 & 0.95 \\
FF & $164 / 16 / 26 / 212$ & 0.911 & 0.891 & 0.90 & 0.97 \\
$\boldsymbol{f}_{\text {cc }}$ & $89 / 15 / 15 / 139$ & 0.663 & 0.903 & 0.75 & 0.65 \\
F $_{\text {II }}$ & $189 / 15 / 1 / 213$ & 0.926 & 0.995 & 0.96 & 0.99 \\
\hline
\end{tabular}

Table 1 provides details on classification performance of these methods. Our experiment shows similar results to those reported results. As we can see, because Compactness measures compact nature of the object rather than roughness, it gives a satisfactory sensitivity $(88.1 \%)$ and a relatively high specificity $(91.5 \%)$ with $A_{z}=0.96$. FD results in the highest sensitivity $(93.5 \%)$ but also the lowest specificity ( $86.8 \%$ ) among these five methods due to its insensitivity to large indentations or protrusions. Fraction Concavity shows a poor sensitivity $(66.3 \%)$ as expected $\left(A_{z}=\right.$ 0.65 ) because it fails to look into the characteristics of structural distortions, such a spicules. FF actually shows satisfactory results in terms of both sensitivity $(91.1 \%)$ and specificity $(89.1 \%)$, but as we can see in the table, after we have changed the shape factor from coordinates to origin distance descriptor, which is the factor we used in our proposed method $\mathrm{F}_{\mathrm{II}}$, the performance is improved in both sensitivity and specificity. It can be observed in Table 1 and Figure 2: the sensitivity and specificity have been improved $1.5 \%$ and $10.4 \%$, respectively; and the area $\left(A_{z}\right)$ under the curve of $\mathrm{F}_{\mathrm{II}}$ is also larger than the one FF yields (0.99 vs. 0.97).
In summary, our proposed irregularity measure, Fourier Irregularity Index, outperforms other approaches with accuracy of $96 \%$ and $A_{z}$ index of 0.99 .

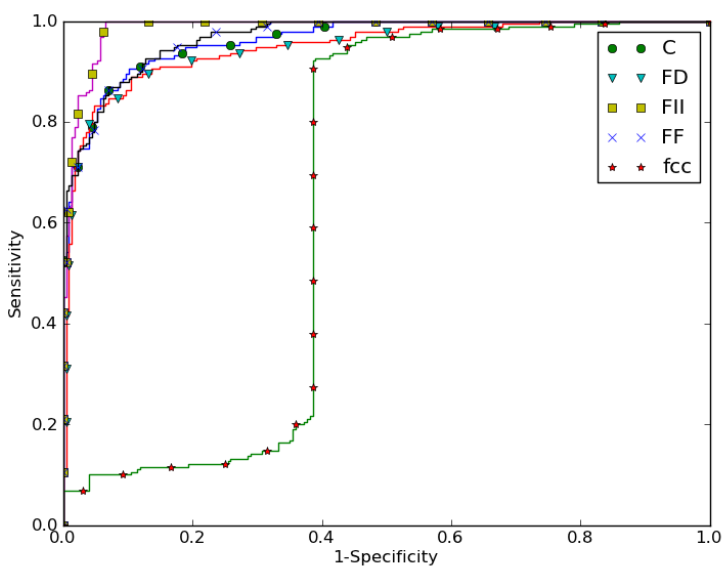

Figure 2 ROC plots for Compactness (C), Fractal Demension (FD), Fourier Factor (FF), Fractional Concavity $\left(f_{c c}\right)$, Fourier Irregularity Index $\left(\mathrm{F}_{\mathrm{II}}\right)$.

However, because of the nature of origin distance descriptor, the constructed sequence cannot always be converted back to the original shape, especially when the descriptor was constructed from a self-intersecting shape. Because our algorithm has a weak capability to capture such complicate factors, the accuracy of it may fluctuate more than expected. Luckily, it is rare and as mentioned, we can bypass it because the medical properties derived from the shape characteristic are important, not the actual shapes. Furthermore, a contour-based analysis method highly depends on its object extraction algorithm. Thus, an ineffective object extraction algorithm could lead the outcome of $F_{\text {II }}$ to uninterruptable results, which is also a challenge in medical image analysis/understanding.

\section{CONCLUSION}

As discussed in this paper, our experimental results show that the proposed approach $\mathrm{F}_{\mathrm{II}}$ achieves malignant/benign classification accuracy of $96 \%$ with $A_{z}$ index of 0.99 . It could be effectively applied to measure irregularity of breast tumor borders. Thus, a helpful reference to diagnose malignancy of tumors could be provided to radiologists by the proposed $\mathrm{F}_{\mathrm{II}}$. It is also possible that a CAD system can benefit in performance improvement from our approach by adopting $\mathrm{F}_{\mathrm{II}}$ as one of classification factors.

\section{ACKNOWLEDGMENTS}

This research was supported by the KCC (Korea Communications Commission), Korea, under the R\&D program supervised by the KCA (Korea Communications Agency), KCA-2012-11911-01108.

\section{REFERENCES}

[1] U.S. Breast Cancer Statistics. (2011). [Online]. Available: http://www.breastcancer.org/symptoms/understand_bc/statist ics.jsp

[2] B. L. D'Orsi CJ, Berg WA, et al., "BI-RADS: Mammography, 4th edition in: D'Orsi CJ, Mendelson EB, Ikeda DM, et al: Breast Imaging Reporting and Data System: ACR BI-RADS - Breast Imaging Atlas, Reston, VA," American College of Radiology, 2003. 
[3] R. Bird, T. Wallace, and B. Yankaskas, "Analysis of cancers missed at screening mammography," Radiology, vol. 184, p. 613, 1992.

[4] E. Lazarus, M. B. Mainiero, B. Schepps, S. L. Koelliker, and L. S. Livingston, "BI-RADS Lexicon for US and Mammography: Interobserver Variability and Positive Predictive Value1," Radiology, vol. 239, pp. 385-391, 2006.

[5] L. Liberman, A. F. Abramson, F. B. Squires, J. Glassman, E. Morris, and D. Dershaw, "The breast imaging reporting and data system: positive predictive value of mammographic features and final assessment categories," American Journal of Roentgenology, vol. 171, pp. 35-40, 1998.

[6] D. Davies and D. Dance, "Automatic computer detection of clustered calcifications in digital mammograms," Physics in Medicine and Biology, vol. 35, p. 1111, 1990.

[7] W. Spiesberger, "Mammogram inspection by computer," Biomedical Engineering, IEEE Transactions on, pp. 213219, 1979.

[8] W. G. Wee, M. Moskowitz, N. C. Chang, Y. C. Ting, and S. Pemmeraju, "Evaluation of mammographic calcifications using a computer program," Radiology, vol. 116, pp. 717$720,1975$.

[9] R. M. Rangayyan, N. El-Faramawy, J. E. L. Desautels, and O. A. Alim, "Measures of acutance and shape for classification of breast tumors," Medical Imaging, IEEE Transactions on, vol. 16, pp. 799-810, 1997.

[10] R. M. Rangayyan, N. R. Mudigonda, and J. E. L. Desautels, "Boundary modelling and shape analysis methods for classification of mammographic masses," Medical \& Biological Engineering \& Computing, vol. 38, pp. 487-496, Sep 2000.

[11] Q. Guo, V. Ruiz, J. Shao, and F. Guo, "A novel approach to mass abnormality detection in mammographic images," 2005.

[12] R. M. Rangayyan and T. M. Nguyen, "Fractal analysis of contours of breast masses in mammograms," Journal of Digital Imaging, vol. 20, pp. 223-237, 2007.

[13] T. K. Lee, D. I. McLean, and M. S. Atkins, "Irregularity index: a new border irregularity measure for cutaneous melanocytic lesions," Med Image Anal, vol. 7, pp. 47-64, Mar 2003.

[14] L. Shen, R. M. Rangayyan, and J. E. L. Desautels, "Application of Shape-Analysis to Mammographic Calcifications," IEEE transactions on medical imaging, vol. 13, pp. 263-274, Jun 1994.

[15] T. K. Lee and E. Claridge, "Predictive power of irregular border shapes for malignant melanomas," Skin Research and Technology, vol. 11, pp. 1-8, 2005.

[16] A. Kikuchi, S. Kozuma, K. Sakamaki, M. Saito, G. Marumo, T. Yasugi, and Y. Taketani, "Fractal tumor growth of ovarian cancer: sonographic evaluation," Gynecol Oncol, vol. 87, pp. 295-302, Dec 2002.

[17] J. W. Cooley and J. W. Tukey, "An algorithm for the machine calculation of complex Fourier series," Math. Comput, vol. 19, pp. 297-301, 1965.

[18] M. Heath, K. Bowyer, D. Kopans, R. Moore, and P. Kegelmeyer, "The digital database for screening mammography," 2000, pp. 212-218.

[19] H. Freeman, "On the Encoding of Arbitrary Geometric Configurations," Electronic Computers, IRE Transactions on, vol. EC-10, pp. 260-268, 1961.

[20] N. A. Obuchowski, "ROC analysis," American Journal of Roentgenology, vol. 184, pp. 364-372, 2005.

[21] G. Zhang, W. Wang, J. Moon, J. K. Pack, and S. I. Jeon, "A review of breast tissue classification in mammograms," presented at the Proceedings of the 2011 ACM Symposium on Research in Applied Computation, Miami, Florida, 2011.

[22] S. LIANG, M. R. RANGARAJ, and J. DESAUTELS, "DETECTION AND CLASSIFICATION OF MAMMOGRAPHIC CALCIFICATIONS," International Journal of Pattern Recognition and Artificial Intelligence, vol. 7, 1993.

[23] H. Alto, R. M. Rangayyan, and J. E. L. Desautels, "Contentbased retrieval and analysis of mammographic masses," Journal of Electronic Imaging, vol. 14, Apr-Jun 2005. 